\title{
The 10th anniversary of the Junior Members and Affiliates of the European Academy of Allergy and Clinical Immunology
}

Chrysanthi L. Skevaki ${ }^{1}$, Paraskevi Maggina ${ }^{1}$, Alexandra F. Santos ${ }^{2,3}$, Rodrigo Rodrigues-Alves ${ }^{4}$, Dario Antolin-Amerigo ${ }^{5}$, Luis Miguel Borrego ${ }^{6,7}$, Isabell Bretschneider ${ }^{8}$, Indre Butiene ${ }^{9}$, Mariana Couto $^{10,11}$, Filippo Fassio ${ }^{12}$, James Gardner ${ }^{13}$, Maria Xatzipsalti ${ }^{14}$, Lilit Hovhannisyan ${ }^{15}$, Valerie Hox $^{16}$, Heidi Makrinioti ${ }^{1}$, Serena E. O’Neil ${ }^{17}$, Gianni Pala ${ }^{18}$, Michael Rudenko ${ }^{19}$, Annalisa Santucci ${ }^{20}$, Sven Seys ${ }^{21}$, Milena Sokolowska ${ }^{22}$, Paul Whitaker $^{23}$ \& Enrico Heffler ${ }^{24}$

\footnotetext{
${ }^{1}$ Allergy Research Laboratories, Second Department of Pediatrics, University of Athens, Athens, Greece; ${ }^{2}$ Department of Pediatric Allergy, Division of Asthma, Allergy \& Lung Biology, King's College London-MRC and Asthma UK Centre in Allergic Mechanisms of Asthma, London, UK; ${ }^{3}$ mmmunoallergology Department, Coimbra University Hospital, Coimbra, Portugal; ${ }^{4}$ Allergy and Clinical Immunology Unit, Hospital do Divino Espírito Santo de Ponta Delgada EPE, Ponta Delgada, Portugal; ${ }^{5}$ Allergy Unit, Immune System Diseases \& Oncology Service, Hospital Universitario Príncipe de Asturias, Alcalá de Henares University, Madrid, Spain; ${ }^{6}$ Immunoallergy Department, Dona Estefânia Hospital, Lisbon, Portugal; ${ }^{7}$ CEDOC, Immunology Department, Faculty of Medical Sciences, NOVA-Universidade Nova de Lisboa, Lisbon, Portugal; ${ }^{8}$ Dept of Paediatric Pneumology and Immunology, Charité Medical School, Berlin, Germany; ${ }^{9}$ Department of Pathology, Pharmacology and Forensic medicine, Faculty of Medicine, Vilnius University, Vilnius, Lithuania; ${ }^{10}$ Immunoallergology Department, Hospital of São João EPE, Porto, Portugal; ${ }^{11}$ Immunology Department, Faculty of Medicine, University of Porto, Porto, Portugal; ${ }^{12}$ Department of Biomedicine, Immunology and Cell Therapies Unit, AOU Careggi, University of Florence, Florence, Italy; ${ }^{13}$ Department of Child Health, Royal Free Hospital, London, UK; ${ }^{14}$ First Department of Pediatrics, 'P \& A Kyriakou' Children's Hospital, Athens, Greece; ${ }^{15}$ Laboratory of Macromolecular Complexes, Institute of Molecular Biology, National Academy of Sciences, Yerevan, Armenia; ${ }^{16}$ Department of Otorhinolaryngology, Head and Neck Surgery, University Hospitals Leuven, Leuven, Belgium; ${ }^{17}$ Krefting Research Centre, Department of Internal Medicine, University of Gothenburg, Gothenburg, Sweden; ${ }^{18}$ Allergy and Immunology Unit, Fondazione Salvatore Maugeri, Institute of Care and Research, Scientific Institute of Pavia, Pavia, Italy; ${ }^{19}$ Clinical Research Group in Fungal Allergens, London, UK; ${ }^{20}$ Allergy and Immunology Clinic, Rimini Infermi Hospital, Rimini, Italy; ${ }^{21}$ Clinical Immunology, Department of Experimental Medicine, Catholic University of Leuven, Leuven, Belgium; ${ }^{22}$ Critical Care Medicine Department, Clinical Center, National Institutes of Health, Bethesda, MD, USA; ${ }^{23}$ Regional Adult Cystic Fibrosis Unit, St James's Hospital, Leeds, UK; ${ }^{24}$ Allergy and Clinical Immunology, Department of Biomedical Sciences and Human Oncology, University of Torino, Turin, Italy
}

To cite this article: Skevaki CL, Maggina P, Santos AF, Alves RR, Antolin-Amerigo D, Borrego LM, Bretschneider I, Butiene I, Couto M, Fassio F, Gardner J, Xatzipsalti M, Hovhannisyan L, Hox V, Makrinioti H, O'Neil SE, Pala G, Rudenko M, Santucci A, Seys S, Sokolowska M, Whitaker P, Heffler E. The 10th anniversary of the Junior Members and Affiliates of the European Academy of Allergy and Clinical Immunology. Pediatric Allergy Immunology 2011 : 22: 754-757.

\section{Keywords}

allergy; clinical immunology; European Academy of Allergy and Clinical Immunology; education; Junior Members and Affiliates.

\section{Correspondence}

Chrysanthi L. Skevaki, Allergy Research Laboratories, Second Department of Pediatrics, University of Athens, 41 Fidipidou str, 11527 Athens, Greece. Tel.: +30 2107776964

Fax: +30 2107777693

E-mail: cskevaki@allergy.gr

Accepted for publication 11 October 2011

DOI:10.1111/j.1399-3038.2011.01238.x

\begin{abstract}
This year is the 10th anniversary of the European Academy of Allergy and Clinical Immunology (EAACI) Junior Members and Affiliates (JMAs). The aim of this review is to highlight the work and activities of EAACI JMAs. To this end, we have summarized all the initiatives taken by JMAs during the last $10 \mathrm{yr}$. EAACI JMAs are currently a group of over 2380 clinicians and scientists under the age of $35 \mathrm{yr}$, who support the continuous education of the Academy's younger members. For the past decade, JMAs enjoy a steadily increasing number of benefits such as free online access to the Academy's journals, the possibility to apply for Fellowships and the Mentorship Program, travel grants to attend scientific meetings, and many more. In addition, JMAs have been involved in task forces, cooperation schemes with other scientific bodies, organization of JMA focused sessions during EAACI meetings, and participation in the activities of EAACI communication platforms. EAACI JMA activities represent an ideal example of recruiting, training, and educating young scientists in order for them to thrive as future experts in their field. This model may serve as a prototype for other scientific communities, several of which have already adapted similar policies.
\end{abstract}


According to futurist Alvin Toffler, 'for society to attempt to solve its desperate problems without the full participation of even very young people is imbecile'. This becomes even more relevant in the context of scientific communities, which are compelled to keep pace with the rapid progress in the biomedical field. The European Academy of Allergy and Clinical Immunology (EAACI) recognized very early the significance of integrating young scientists in the ongoing evolution of research and clinical practice.

\section{History and benefits of Junior Members and Affiliates (JMAs)}

The JMAs of the EAACI are now a group of over 2380 clinicians and scientists under the age of $35 \mathrm{yr}$. Initially founded in 2001, the group aims at supporting the education of the Academy's younger members, as well as their participation in, and influence on, EAACI activities. This year, JMAs celebrate their 10th anniversary and have added even more activities on their agenda.

Junior Members and Affiliates enjoy a number of benefits as members of the world's most active allergy and clinical immunology association. These include free membership and online access to the official EAACI journals (Allergy and Pediatric Allergy \& Immunology), the possibility to apply for Research and Clinical Fellowships, and travel grants to attend EAACI Annual Congresses but also smaller Focused Meetings and Allergy Schools. Indeed, according to the 2010 and 2011 online questionnaires addressing JMAs, travel grants represented the only funding opportunity to attend the aforementioned meetings for the majority of juniors who took part in the survey (1). Junior membership in the Academy also provides the inestimable possibility to become actively involved in all of its activities by applying for a position in the JMA Working Group (WG). The JMA WG is the representative body of EAACI JMAs, which until recently was composed of a webmaster, one representative from each of the five EAACI sections and a chairperson (Table 1). As of this year, the WG has expanded to also include one representative from each of the EAACI Interest Groups (Table 2).

\section{JMA sessions at EAACI meetings}

Since the establishment of JMAs in 2001, numerous initiatives have increased their participation in the Academy's life.
Table 2 Junior Members and Affiliates Working Group Board Members from 2011 to 2013

\begin{tabular}{ll}
\hline & 2011-2013 \\
\hline Chairperson & Enrico Heffler \\
Asthma S Rep & Sven Seys \\
Dermatology S Rep & Lilit Hovhannisyan \\
ENT S Rep & Valerie Hox \\
Immunology S Rep & Milena Sokolowska \\
Pediatrics S Rep & Alexandra Santos \\
Webmaster & Rodrigo Rodrigues Alves \\
Aerobiology and Pollution IG Rep & Indre Butiene \\
Allergy, Asthma and Sport IG Rep & Mariana Couto \\
Allergy Diagnosis IG Rep & Filippo Fassio \\
Allied Heath IG Rep & James Gardner \\
Drug Allergy IG Rep & Paul Whitacker \\
Food Allergy IG Rep & Annalisa Santucci \\
Functional Genomics and & Serena O'Neil \\
Proteomics IG Rep & \\
Immunotherapy IG Rep & Paraskevi Maggina \\
Infections and Allergy IG Rep & Heidi Makrinioti \\
Insect Hypersensitivity IG Rep & Dario Antolin-Amérigo \\
Occupational Allergy IG Rep & Gianni Pala \\
\hline
\end{tabular}

S, Section; IG, Interest Group; Rep, Representative; ENT, Ear, Nose, Throat.

Thus, Juniors now enjoy a series of sessions during the Annual Congress that are targeted toward their needs as physicians and basic scientists at the dawn of their career. The JMA Poster Session is co-chaired by one junior and one senior EAACI member and provides prizes for the most outstanding presentations. The latter also holds for the best three of the 10 short presentations in the JMA Case Reports Session. The JMA Scientific Symposium features cutting edge science presented by JMAs, while the JMA Educational Session aims at providing support to young scientists in regard to common everyday challenges in their professional lives. The JMA Business Meeting and Social Event are the platform for the strategic programing of the group and networking. Apart from the Annual Congress, JMAs have also organized oral abstract symposia and other scientific symposia in the context of Focused Meetings. Furthermore, Allergy Schools organized by the Academy are activities addressing mainly the younger membership, with the opportunity to receive travel grants in conjunction with abstract

Table 1 Junior Members and Affiliates Working Group Board Members from 2001 to 2011

\begin{tabular}{|c|c|c|c|c|c|}
\hline & 2001-2003 & 2003-2005 & 2005-2007 & 2007-2009 & 2009-2011 \\
\hline Chairperson & Susanna Olsson & Philippe Gevaert & Ulrike Raap & Luis Miguel Borrego & Chrysanthi Skevaki \\
\hline Asthma S Rep & Bart Lambrecht & Gert-Jan Braunstahl & David Groneberg & David Groneberg & Enrico Heffler \\
\hline Dermatology S Rep & Ulrike Raap & Ulrike Raap & Elena Borzova & Elena Borzova & Lilit Hovhannisyan \\
\hline ENT S Rep & Philippe Gevaert & Peter Hellings & Michael Rudenko & Michael Rudenko & Michael Rudenko \\
\hline Immunology S Rep & Stefano Del Giacco & Stefano Del Giacco & Marcin Kurowski & Marcin Kurowski & Milena Sokolowska \\
\hline Webmaster & Ioana Agache & Ioana Agache & Chrysanthi Skevaki & Chrysanthi Skevaki & Rodrigo Rodrigues Alves \\
\hline
\end{tabular}

S, Section; Rep, Representative; ENT, Ear, Nose, Throat. 
submission. JMA WG members who attend these schools write reports, which are published on http://www.eaaci.net and the EAACI Newsletter.

\section{The pediatric perspective}

From the Pediatrics Section perspective, the JMA representative is naturally involved in planning the sessions proposed by the section for inclusion in the Scientific Program of the Annual Congress, as well as in the evaluation of related abstracts. JMAs have been involved in the 2005 and the 2007 joint meeting of the Pediatrics Sections of the EAACI and the European Respiratory Society (ERS), held in Prague, Czech Republic, and Lisbon, Portugal, respectively. More specifically, JMAs have participated in the Local Organizing Committee and its preparations but also included a scientific symposium with EAACI Juniors as speakers. JMAs were also systematically involved in a number of sessions of the 2009 Pediatric Allergy and Asthma Meeting held in Venice, Italy. What is more to add, research activities of the JMA Pediatrics Section Representatives but also of other JMAs belonging to the Pediatrics Section are regularly highlighted through publications in the Academy's official journals (2-4).

\section{Education and training opportunities for JMAs}

The European Academy of Allergy and Clinical Immunology, in collaboration with the UEMS, have prepared an examination on Allergology/Clinical Immunology, which has been held annually since 2008 during the Annual Congress of the Academy. This initiative aims at further improving the standards of the specialty by harmonizing the educational level throughout Europe. JMAs have been actively involved in its preparation and evaluation since its inception, through the inclusion of a JMA representative in the EAACI Exam Task Force (TF), which has now been transformed to a committee. The exam serves as a self-evaluation tool and is expected to facilitate the mobility of young people trained in Allergology and Clinical Immunology across the continent, while JMAs are entitled to waived exam registration fees.

Juniors are now represented within several other TFs as well, such as the revised 'PRACTALL on Pediatric Asthma' and 'Pediatric Rhinitis', a joint TF of the Pediatrics, Asthma, and ENT Sections.

The EAACI Clinical and Research Fellowship Awards are another important achievement targeting the younger members of the Academy. These awards aim at spreading Allergy and Clinical Immunology in Europe, supporting research and training of JMAs and highlighting their work through increased publications. Additionally, Clinical and Research Fellowship Awards provide the means for increasing the mobility of young researchers within Europe and spreading the implementation of new techniques.

Quite recently, the Mentorship Program (MP), a new initiative developed by the JMA WG, was officially launched in the context of EAACI Istanbul 2011. The MP plans to ensure ongoing education for young scientists and to enhance JMA capacities as professionals, thus promoting the objectives of our field. This is a strategy aimed at building members' skills and career and taking a strong profession into the future. The MP is expected to improve knowledge transfer of experienced professionals to Juniors, increase JMAs' appreciation of and orientation in the field, and promote opportunities for JMAs to work closely with a faculty mentor for the development of a paper for presentation at an academic conference and/or for publication. Following the first open call in 2011, 87 potential mentors were enrolled, who are all renowned members of the Academy, while 36 of 41 applications submitted by JMAs were accepted in the MP.

\section{JMAs in the communication field}

In the communications field, JMAs have been included in the Editorial Boards of the http://www.eaaci.net as well as the http://www.allergynews.com and contribute regularly to their online content. Also, several articles and reports of the EAACI Newsletter are composed by JMAs. Very importantly, a new collaboration between JMAs and the Pediatric Allergy and Immunology (PAI) journal has been recently initiated (5). Core elements of this alliance are the selection of 'Must read papers' (6) from each PAI issue for medical residents and other young scientists in the field, the opportunity provided to JMAs to submit 'hot topic reviews' in cooperation with their mentors for publication in the PAI journal, as well as the participation of JMAs who are highly active in research and more advanced in their career in the PAI editorial process by acting as reviewers.

\section{Collaboration schemes within and beyond the academy}

JMAs currently participate in other EAACI bodies as well, such as the CME Committee but also in non-EAACI bodies, such as the Allergy UEMS Section and Board. Moreover, they have cultivated collaborations with respective sectors of other related scientific societies, such as the Fellows in Training of the American Academy of Allergy, Asthma and Immunology (AAAAI) and the Juniors of the Federation of Clinical Immunology Societies (FOCIS) and of the European Rhinology Society.

\section{Conclusion}

It becomes evident that the EAACI JMAs have evolved tremendously over the past decade. This is of particular significance for an association of clinicians, researchers, and allied health professionals, dedicated to improving the health of people affected by allergic diseases. Today's JMAs are tomorrow's top scientists and opinion leaders. Thus, JMA activities and opportunities targeting this community represent excellent approaches for their continuous education, training, and preparation as future allergy and clinical immunology experts. Join the EAACI JMAs now and become part of one of the most dynamic groups of scientists in the world. 


\section{References}

1. Skevaki CL, Rudenko M, Xatzipsalti M, et al. Junior Member Working Group audit of junior members' preferences and expectations from EAACI membership and JMA scientific activity at EAACI Congresses and EAACI Allergy Schools. Allergy 2010: 65 (Suppl. 92): 145.

2. Maggina $\mathrm{P}$, Makrinioti $\mathrm{CN}$, Papaevangelou $\mathrm{V}$, et al. IL-8: a possible marker of increased susceptibility of infants to bronchiolitis and subsequent recurrent episodes of wheezing one year later. Allergy 2011: 66 (Suppl. 94): 73.

3. Muraro A, Clark A, Beyer K, et al. The management of the allergic child at school: EAACI/GA2LEN Task Force on the allergic child at school. Allergy 2010: 65: 6819.

4. Santos A, Dias A, Pinheiro JA. Predictive factors for the persistence of cow's milk allergy. Pediatr Allergy Immunol 2010: 21: 1127-34.

5. Wahn U. PAI joins forces with young allergy researchers. Pediatr Allergy Immunol 2011: 22: 546.

6. Caubet JC, Pichler WJ, Eigenmann PA. Educational case series: mechanisms of drug allergy. Pediatr Allergy Immunol 2011: 22: 559-67. 